\title{
Subjective perception of body sway
}

\author{
Marco Schieppati, Elena Tacchini, Antonio Nardone, Jessica Tarantola, Stefano Corna
}

\begin{abstract}
Objectives and method-The relation between body sway recorded through a stabilometric platform and the subjective report of steadiness was studied in 20 young and 20 elderly subjects and 20 neuropathic and 20 parkinsonian patients standing upright. The trials were performed under two stances (feet apart, feet together) and two visual conditions (eyes open, eyes closed). At the end of each trial, subjects scored their performance on a scale from 10 (complete steadiness) to 0 (fall).
\end{abstract}

Results-In all subjects, independently of the stance conditions, the larger the body sway the smaller the reported score. The function best fitting this relation was linear when sway was expressed on a logarithmic scale. The scoring reproducibility proved high both within and across subjects. Despite the different body sways and scores recorded under the different visual and postural conditions (eyes closed >eyes open, feet together $>$ feet apart) in all groups of subjects and patients, the slopes of the relations between sway and score were broadly superimposable. In the normal subjects, the scores were slightly higher during eyes open than eyes closed trials for corresponding body sways. This was interpreted as a sign of perception of greater stability when vision was allowed. Parkinsonian patients swayed to a similar extent as normal subjects, and their scores were accordingly similar, both with eyes open and eyes closed. Neuropathic patients swayed to a larger extent than normal subjects, and their scores were matched appropriately. Although the slope of their relation with eyes closed was not different from that of normal subjects, with eyes open it was steeper and similar to that with eyes closed, suggesting that these patients did not feel more stable when they could take advantage of vision. Conclusions-The subjective evaluation of body sway, irrespective of stance condition, age, neuropathy, and basal ganglia disease, reflects the actual sway, and is inversely proportional to the logarithm of the sway value. The remarkable similarity of the relation between score and sway across the various groups of subjects with eyes closed indicates a common mode of sway evaluation, possibly based on integration of several sensory inputs. All groups except neuropathic patients seem to take advantage of the redundancy of the inputs. Basal ganglia integrity does not seem to have a role in the evaluation of sway.

(F Neurol Neurosurg Psychiatry 1999;66:313-322)

Keywords: balance; perception; stabilometry

It is not an uncommon experience for people involved in research on the control of posture and movement to find patients reporting postural instability or unsteadiness but giving normal stabilographic recordings when standing at ease on a dynamometric platform. This is the case with parkinsonian patients, in whom body sway area and sway path are often within the normal range ${ }^{1-4}$ despite the reported (and clinically assessed) postural insecurity of these patients. Therefore, the question can be asked whether problems in the perception or evaluation of postural instability may lead to frightened stance, or unsteadiness of gait, or both. On the other hand, people affected by peripheral neuropathies can hardly be clustered in a single group according to the stabilographic recordings and to the self reported feeling of unsteadiness. In fact, they report disturbing unsteadiness or complain of hazardous stance, and are prone to falls ${ }^{5}$; but, in some cases they do not show abnormally large stabilographic recordings, ${ }^{6}$ although in other cases they do.

There is as yet no indication of congruence between absolute body sway and perception of it. An increasing focus on qualitative investigation in medicine brings with it a tendency to model qualitative research along quantitative lines. ${ }^{8}$ Examples are self evaluation scales or inventories, designed to be filled in by the subject or patient without the intervention of the examiner. ${ }^{9}$ Such scales would seem particularly useful in approaching patients reporting dizziness or disturbance of balance. Relations between components of balance function and self perceived dizziness handicap have already been assessed in vestibular patients and elderly subjects. ${ }^{10}{ }^{11}$ However, a scale bringing together the perception of stability during a postural task, such as quiet upright stance, and the real steadiness recorded with a stabilometric platform is still lacking.

The aim of this investigation was to assess the capability, if any, of normal subjects to perceive and evaluate their own stability during short periods of quiet stance under different sensorial conditions, and to determine whether a self evaluation score could be a relevant variable for further use. To this end, the congruity between the subjective reports and objective stabilometric findings was analysed, using a simple subjective scale of steadiness. The effect of aging on the perception of sway was also 
Table 1 Clinical and electrophysiological characteristics of the neuropathic patients

\begin{tabular}{lllllll}
\hline Patient No & Sex/Age & Type of neuropathy & Equil & CV Sur & CV CP & CV TP \\
\hline 1 & M 77 & Diabetic & 23 & NM & 34 & 34 \\
2 & F 76 & Idiopathic sensorimotor & 23 & NM & 41 & 48 \\
3 & F 42 & HMSN I & 20 & NM & 23 & 12 \\
4 & M 76 & Idiopathic sensorimotor & 24 & NM & 15 & 8 \\
5 & M 54 & Idiopathic sensorimotor & 13 & NM & 14 & NM \\
6 & M 46 & HMSN II & 18 & NM & 29 & 35 \\
7 & M 61 & HMSN I & 26 & NM & 24 & 22 \\
8 & F 73 & Idiopathic sensorimotor & 11 & NM & 33 & 36 \\
9. & F 54 & Diabetic & 22 & 39 & 38 & 33 \\
10 & M 29 & HMSN I & 26 & NM & 23 & 21 \\
11 & F 34 & HMSN II & 26 & NM & 38 & 36 \\
12 & F 70 & Diabetic & 23 & NM & 36 & 32 \\
13 & F 72 & Idiopathic sensory & 18 & NM & 46 & 43 \\
14 & F 70 & Idiopathic axonal & 24 & 50 & 49 & 41 \\
15 & M 55 & Idiopathic sensorimotor & 23 & 38 & 33 & 37 \\
16 & M 63 & Diabetic & 16 & NM & 22 & 31 \\
17 & F 46 & Idiopathic sensorimotor & 23 & 40 & 34 & 32 \\
18 & M 69 & Diabetic & 23 & NM & 30 & 31 \\
19 & M 73 & Diabetic & 24 & NM & 35 & 33 \\
20 & F 72 & Diabetic & 23 & NM & 40 & 42
\end{tabular}

HMSN=hereditary motor and sensory neuropathy (type I or II); NM=not measurable; equil=Tinetti score for equilibrium $(\max 26)^{13} ; \mathrm{CV}=$ nerve conduction velocity $(\mathrm{m} / \mathrm{s})$; sur=sural nerve; $\mathrm{CP}=$ common peroneal nerve; $\mathrm{TP}=$ tibialis posterior nerve.

assessed in a group of normal aged subjects. Further, because proprioception from lower limbs is a key feedback which subjects rely on when standing with eyes closed, ${ }^{7}$ and as basal ganglia have a pivotal role in balance control, ${ }^{12}$ we assessed the possible influence of altered proprioception and basal ganglia disorder on the correct perception of body sway in one group of neuropathic and one of parkinsonian patients.

\section{Methods}

SUBJECTS

Twenty young, healthy subjects (five men and 15 women, mean age 30.8 , range 21 to 48 years), 20 normal elderly subjects (seven men and 13 women, mean age 65.8 , range 54 to 82 years), 20 neuropathic patients ( 10 men and 10 women, mean age 61.7 , range 29 to 77 years), and 20 idiopathic parkinsonian patients (nine men and 11 women, mean age 68.5, range 43 to 79 years,) were selected for testing. Tables 1 and 2 summarise the relevant data of the two patient groups. The local ethics committee approved the use of the experimental procedure. All subjects gave their informed consent, although the specific aims of the experiment were not conveyed.

\section{ELECTROPHYSIOLOGICAL AND FUNCTIONAL}

TESTING

Several days before the balance assessment sessions, neuropathic patients underwent separate sessions in which the maximal conduction velocity of sural, tibialis posterior, and common peroneal nerves was measured. The findings of these tests are summarised in table 1. For the sake of simplicity, as the polyneuropathy was broadly symmetric, the values reported in the table are the mean of the responses from both sides. The performance oriented evaluation of balance and gait ${ }^{13}$ was also administered to the patients by an expert physiotherapist at the end of the experimental session: the scores reported in the table refer only to the balance portion of this evaluation.

\section{BALANCE ASSESSMENT}

The three components of the force acting on each of the four transducers of a dynamometric Kistler (type 9281B) platform were converted from analog to digital (sampling rate $10 \mathrm{~Hz}$ ) and fed into a computer. The following variables were computed off line: (1) the position of the instantaneous centre of foot pressure, (2) the projection of the CFP on the

Table 2 Summary of some clinical features of the parkinsonian patients

\begin{tabular}{|c|c|c|c|c|c|c|c|c|c|}
\hline Patient No & Sex/Age & $\operatorname{Dur}(y)$ & Drug & $m g / d a y$ & $H-Y$ & $W$ total & W Brad & W Rig & W Trem \\
\hline \multirow[t]{2}{*}{1} & F 65 & 2 & LDI & 750 & 2 & 5 & 1 & 1 & 0 \\
\hline & & & LDI-R & 375 & & & & & \\
\hline 2 & F 77 & 4 & LDI & 500 & 1 & 4 & 2 & 1 & 0 \\
\hline 3 & F 62 & 2 & LDI & 450 & 1 & 6 & 1 & 1 & 0 \\
\hline \multirow[t]{2}{*}{4} & M 64 & 2 & LDI & 375 & 3 & 9 & 1 & 1 & 1 \\
\hline & & & Amantad & 100 & & & & & \\
\hline 5 & M 74 & 1 & LDI & 175 & 3 & 6 & 1 & 1 & 1 \\
\hline 6 & M 64 & 4 & LDI & 550 & 3 & 7 & 1 & 1 & 2 \\
\hline \multirow[t]{3}{*}{7} & M 70 & 10 & LDI & 500 & 3 & 17 & 3 & 2 & 2 \\
\hline & & & Orphenad & 150 & & & & & \\
\hline & & & Pergolide & 0.75 & & & & & \\
\hline 8 & F 79 & 1 & Untreated & & 3 & 6 & 1 & 0 & 1 \\
\hline 9 & M 57 & 1 & Untreated & & 2 & 5 & 1 & 0 & 1 \\
\hline \multirow[t]{3}{*}{10} & M 72 & 5 & LDI & 575 & 2 & 7 & 1 & 1 & 2 \\
\hline & & & LDI-R & 500 & & & & & \\
\hline & & & Orphenad & 100 & & & & & \\
\hline \multirow[t]{3}{*}{11} & F 79 & 18 & LDI & 450 & 4 & 19 & 3 & 1 & 0 \\
\hline & & & LDI-R & 500 & & & & & \\
\hline & & & Selegiline & 2.5 & & & & & \\
\hline 12 & F 74 & 3 & LDI & 175 & 3 & 12 & 2 & 1 & 0 \\
\hline 13 & F 65 & 1 & LDI & 175 & 2 & 4 & 1 & 1 & 1 \\
\hline \multirow[t]{2}{*}{14} & F 73 & 16 & LDI & 500 & 4 & 15 & 2 & 2 & 0 \\
\hline & & & LDI-R & 375 & & & & & \\
\hline \multirow[t]{2}{*}{15} & M 76 & 5 & LDI & 300 & 2 & 6 & 1 & 0 & 0 \\
\hline & & & LDI-R & 375 & & & & & \\
\hline \multirow[t]{4}{*}{16} & F 68 & 8 & LDI & 450 & 3 & 9 & 2 & 1 & 1 \\
\hline & & & LDI-R & 250 & & & & & \\
\hline & & & Selegiline & 5 & & & & & \\
\hline & & & Bornaprine & 8 & & & & & \\
\hline 17 & M 63 & 0.5 & Untreated & & 2 & 7 & 2 & 1 & 0 \\
\hline \multirow[t]{2}{*}{18} & M 43 & 13 & LDI & 550 & & & & & \\
\hline & & & LDI-R & 125 & 3 & 4 & 1 & 1 & 0 \\
\hline 19 & F 74 & 2 & Untreated & & 2 & 4 & 1 & 0 & 0 \\
\hline 20 & F 71 & 3 & LDI & 375 & 2 & 12 & 2 & 2 & 0 \\
\hline
\end{tabular}

Dur=Duration; H-Y=Hoen and Yahr score; W=Webster score; Brad=bradykinesia; Rig=rigidity; Trem=tremor; LDI=levodopa DOPA decarboxylase inhibitors; $\mathrm{R}=$ slow release; Amantad=amantadine; Orphenad=orphenadrine. 
A
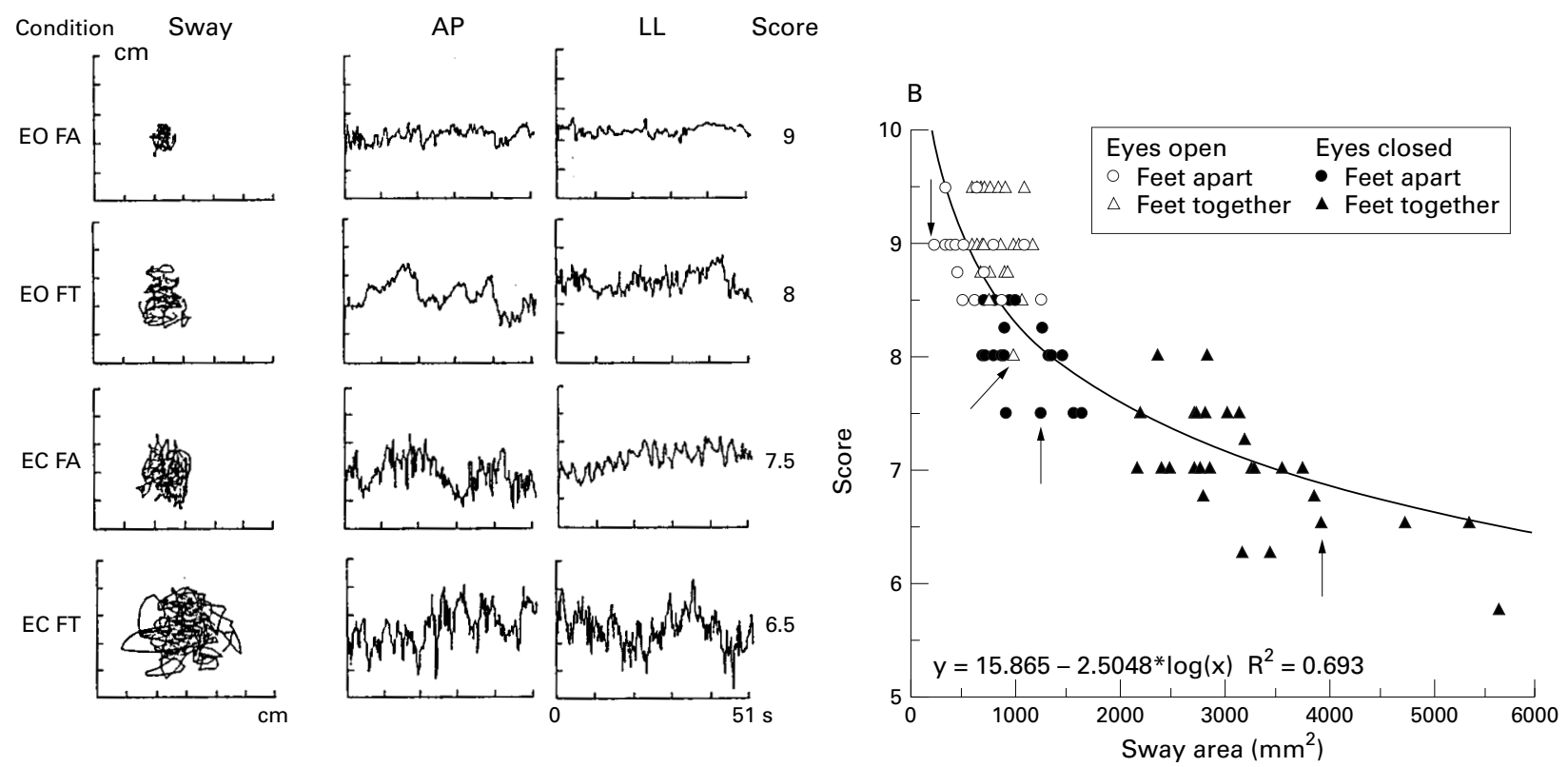

Figure 1 (A) Stabilographic recordings of a representative normal, young subject during quiet stance (eyes open, eyes closed, feet apart, feet together). The stabilograms (sway) and the projections of the displacement of centre of foot pressure on the anteroposterior (AP) and laterolateral (LL) axes are plotted; the numbers are the self evaluation score attributed to the corresponding trials. There is a progressive decrease in the subjective score with the increase of body sway. (B) Relation between subjective scores and sway areas in the same subject. Arrows point to the trials depicted in the left panel. The data points are best fitted through a logarithmic model.

sagittal and frontal planes, (3) the mean position of centre of foot pressure during the trial, ${ }^{14}(4)$ the sway path, or distance covered during the trial by the moving centre of foot pressure; (5) the sway area, or surface swept during the trial by the shift of the line joining the mean centre of foot pressure to the moving centre of foot pressure. ${ }^{15}$

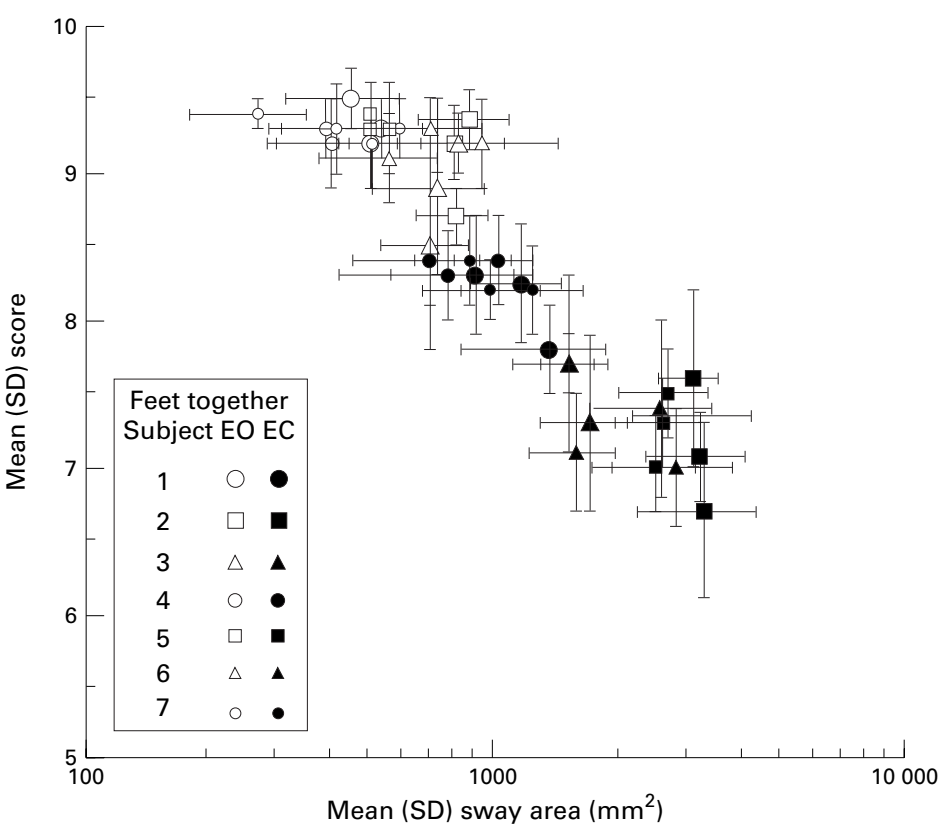

Figure 2 Reproducibility of the subjective scoring of body sway. Each symbol is the mean (SD) of all trials performed with eyes open or closed under the feet together condition by the seven subjects, who participated in three experimental sessions each. Clustering of the data indicates the good reproducibility of the scoring of body sway in each subject, under both visual conditions.
EXPERIMENTAL SESSIONS

Subjects stood upright barefoot on the platform. They were asked to stand still with their arms by their sides. Young normal subjects participated in two sessions. In each session they performed a series of 20 stance trials, alternately with eyes open when they looked at a target placed at eye level about $50 \mathrm{~cm}$ in front of them, and with eyes closed. Each trial lasted 51 seconds. Feet were spaced $10 \mathrm{~cm}$ apart in the first session (feet apart), and kept together (feet together) in the second session, which took place on a different day. Seven of the young normal subjects participated in separate sessions, on three different days, to test reproducibility. Elderly subjects and patients participated in one session only, in which they performed respectively five and three trials for each condition. Total session time was about 30 minutes, including periods during which subjects were allowed to sit to avoid sole soreness or exhaustion, and minimise the effect of close repetition of trials. ${ }^{16}$

At the end of each trial, subjects were asked to evaluate their subjective feeling of the quality of steadiness throughout that particular trial. The evaluation took the form of a score, ranging from 0 (worst) to 10 (best). Half points and quarter points were allowed, to distinguish trials with small differences. To give a reference, at the beginning of the session each subject was invited to stand in two postures corresponding to extremes of the range of stability: (1) with eyes open, feet apart, grasping a solid bar in front of them; this was judged by the subjects as a very stable condition, to which 10 should be attributed, (2) with eyes closed and no support, in a one leg stance condition: this could not be maintained for extended periods of time, and was judged as a very unstable condition meriting 
Table 3 ICC of the score and within subject $C V$ obtained in seven subjects tested on three different days each

\begin{tabular}{llllll}
\hline & & \multicolumn{5}{l}{ (B) Within subject $C V(\%)$} \\
\cline { 3 - 6 } Subject & (A) ICC & 1st & 2nd & 3rd & Mean \\
\hline 1 & 0.96 & 7.61 & 8.09 & 7.36 & 7.68 \\
2 & 0.98 & 12.4 & 13.8 & 19.9 & 15.3 \\
3 & 0.91 & 10.0 & 11.4 & 8.84 & 10.1 \\
4 & 0.88 & 5.98 & 6.41 & 6.11 & 6.17 \\
5 & 0.94 & 8.4 & 5.65 & 6.26 & 6.77 \\
6 & 0.99 & 6.17 & 6.97 & 6.67 & 6.60 \\
7 & 0.90 & 13.58 & 14.57 & 12.57 & 13.57 \\
\hline
\end{tabular}

ICC=intraclass correlation coefficient; CV $(\%)=$ coefficient of variation; 1st, 2nd, and 3rd = experimental sessions.

a very low score; in the case of the subjects lowering the other leg to avoid a fall, a 0 score should be attributed to the trial. All subjects and patients understood the instruction well.

DATA ANALYSIS

The stabilometric data were plotted to see if they were normally distributed. As this was apparently not the case, sway area distribution was tested by means of the KolmogorovSmirnov (K-S) test, separately for each visual and postural condition and for each of the four groups of subjects. The test was significant under all circumstances; therefore, the data were normalised by means of a logarithmic transformation. The transformed data were indeed normally distributed (K-S test not significant), which allowed us to use parametric analysis of the data.

To test the effect of the session on the subjective scores given by each of the seven normal subjects who underwent three sessions for reproducibility, we divided the whole range of sway areas recorded for each subject in each session (under the feet together condition and regardless of eyes open or eyes closed) into four equally spaced blocks. The mean scores attributed to the sway variables falling within each block were then calculated and compared across blocks and sessions by means of the analysis of variance (ANOVA), and the intraclass correlation coefficient (ICC) was obtained.

Values of ICC above 0.75 are generally taken to represent good reliability; for most clinical measurements, reliability should exceed 0.90 to ensure reasonable validity. ${ }^{17} 18$ Therefore, ICC values above 0.90 were considered as an index of the capacity of subjects to attribute largely corresponding scores to similar body sways produced in separate sessions.

To test whether there were differences between the 20 young normal subjects in their subjective reports, the within and between subject coefficient of variability of the score was considered. The first was obtained on the basis of the data (feet together, eyes open and eyes closed) of each subject, the second was calculated on the basis of data of all subjects pooled together.

For all the groups of normal subjects and patients, the relation between score (ordinate) and the logarithm of sway variables (abscissa) was described by the equation of the line best fitting the data. This procedure was followed for both the individual data and the averaged data from repeated trials of all subjects. The difference in the slope of the best fit lines obtained under the various experimental conditions was assessed by means of the analysis of variance (ANOVA) of the linear regressions. This yielded a set of data to be entered in the Student's $t$ test formula. ${ }^{19} 20$

To make inferences on the possible interval property of the subjective scale, it was important to know not only whether the slope of the assumed linear regression was significant, but also whether there was any reason to doubt the basic assumption of the linearity of the regression. Therefore, a test for linearity was applied to the data (feet together, eyes open, and eyes closed, separated in equally spaced blocks, or arrays) obtained in the seven subjects who underwent several trial repetitions; for each subject, linearity of the regression was assumed when the $F_{2}$ value $^{20}$ was not significant.

To test the significance of the differences between the mean sway areas recorded in the four groups of subjects, under the four different stance conditions, a multifactorial ANOVA was used, followed by the Newman-Keuls post hoc test.

\section{Results}

SWAY AND SCORE

Relatively wide variations were found in the stance performances of normal subjects. As expected, variability was much larger with feet together than feet apart, and with eyes closed than open, as were the absolute measures of sway (sway area and sway path). There was also a rather large scatter of body sway values across trials performed under the same conditions by each subject. Further, the various subjects' mean sway values were scattered over a relatively wide range. These variations were indeed welcome prerequisites for a reliable matching between subjective scoring and instrumental outputs.

Figure 1A shows an example of the findings obtained in one representative subject, standing under feet together and feet apart conditions, both with eyes open and eyes closed. The effect of the different conditions is evident in the stabilograms (sway), and in the traces representing the projection of the instantaneous centre of foot pressure on the anteroposterior and laterolateral plane during the entire trial. The subjective scores attributed to these performances are reported in the right hand column. The data from all the trials produced by this subject are summarised in the right panel: sway areas (abscissa) ranged from 200 to $5700 \mathrm{~mm}^{2}$, and the corresponding subjective scores from 9.5 to 5.75 . The line best fitting the scores and sway area points was logarithmic. In other words, the scores did not worsen proportionally to the increase in sway.

Normalisation of the sway area values by logarithmic transformation rectified the relationship. The regression was linear $\left(F_{2}=0.3,2\right.$ and $43 \mathrm{df}$, NS; see methods). This was true for six of the seven normal subjects, who participated in the reproducibility study; in the seventh, $\mathrm{F}_{2}$ was barely significant. 

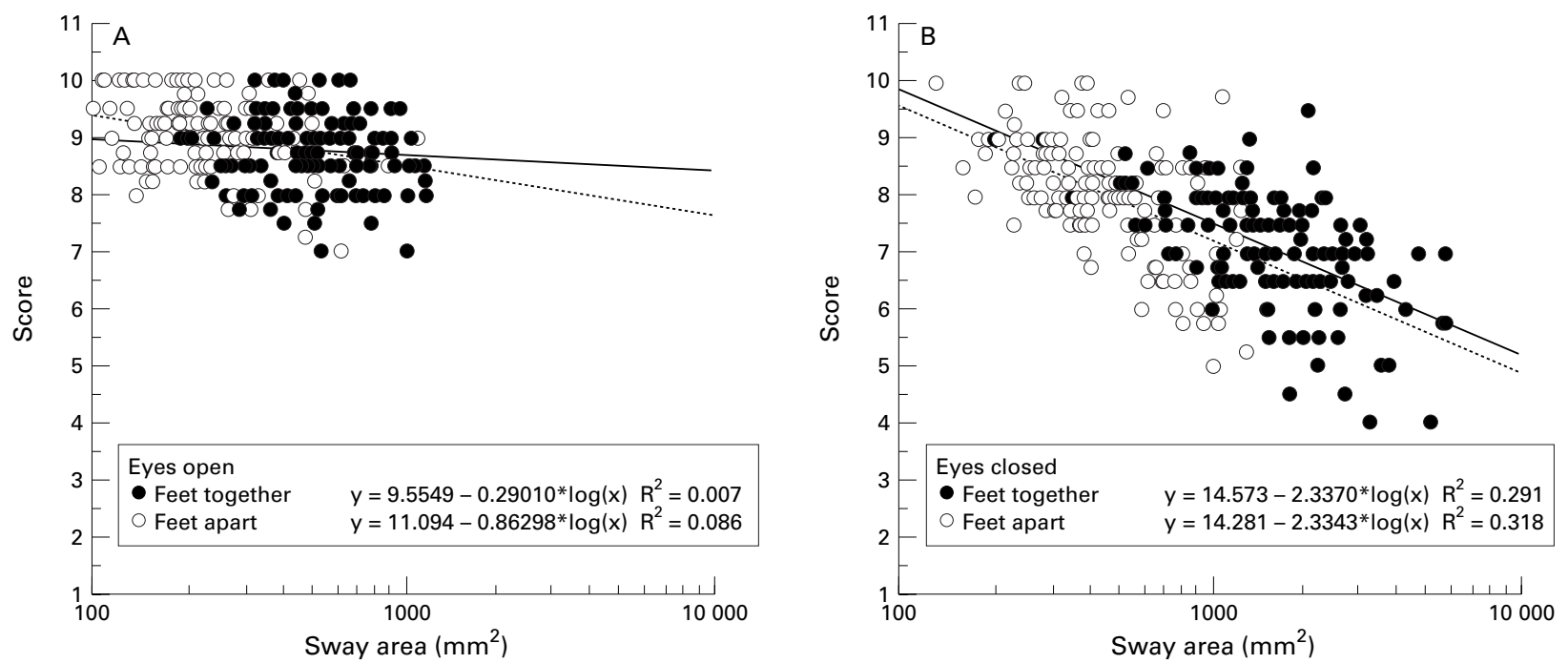

Figure 3 Relation between subjective scores and sway areas (logarithmic scale in the abscissa) for all single trials of all young subjects. A progressive decrease of the score takes place as sway increases, regardless of the stance condition. This effect is greater under eyes closed than eyes open condition. In each panel, the lines best fitting the data points obtained under both stance conditions (feet together, continuous line; feet apart, dotted line) are almost superimposable.

REPRODUCIBILITY OF SCORING

This study was conducted in seven young normal subjects to ascertain that the scores given in separate sessions by the same subject for similar sway areas were similar across the whole span of sway areas obtained over the various trials. To this end, we arbitrarily divided the range of sway areas into four segments and separately analysed the scores given to the sway areas falling within each segment. The ICC (table $3 \mathrm{~A}$ ) proved to be well above 0.90 for each subject except one. Furthermore, for the same subjects, the coefficient of variation (CV) was very small and very similar across the three sessions (table $3 \mathrm{~B}$ ). In figure 2, each symbol corresponds to the mean value of sway area and score produced by each subject (feet together) across the different sessions. Equal symbols are very close together, indicating a good average reliability of the subjective scoring of body sway, both with eyes open (open symbols) and eyes closed (filled symbols), in spite of a sizeable variability around the mean value.

When the data from all 20 normal subjects were pooled, the between subject CV (14.4\%) were very similar to the mean of the within subject CV (13.3\%). This means that different subjects attributed a similar score to corresponding sway areas, and that the variance between normal subjects was negligible, and similar to the variance within each subject. This is a prerequisite for pooling together the data of all subjects as in fig 3 .

\section{YOUNG SUBJECTS}

Figure 3 is the result of pooling together all the single data points obtained in each young normal subject after logarithmic transformation of the values of the sway areas (the data obtained under the eyes open and eyes closed conditions have been separated into two parts, A and B respectively). (1) Straight lines best fitted the
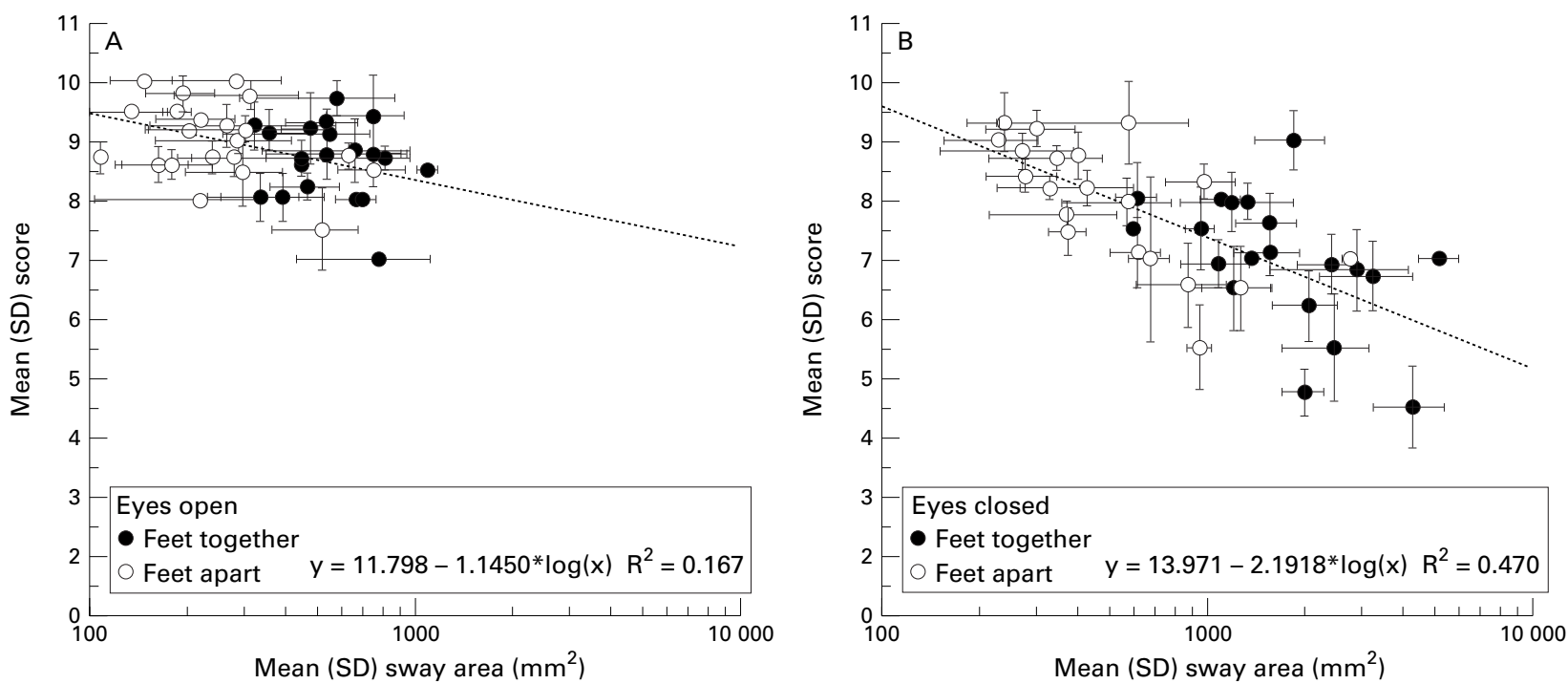

Figure 4 Relation between mean score (SD) and mean sway area (logarithmic scale in the abscissa) in the young subjects. A similar variance of scores and sways exists in all subjects, under all visual and stance conditions. A single line is fitted through the data obtained under each visual condition. The slopes of the best fit lines in $A$ and $B$ are significantly different. 


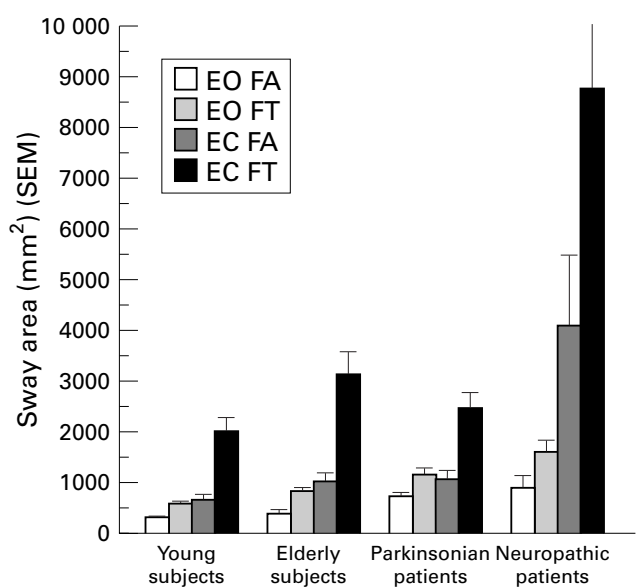

Figure 5 Comparison of the body sway areas across the groups studied. In all groups, each column is the average of sway area (SEM) obtained from 20 subjects.

transformed data under both feet apart and feet together conditions, within each visual condition; (2) there was no significant difference between the lines best fitting the feet apart and feet together data, under each visual condition; (3) There was a difference $(p<0.0005)$ between the lines fitting the eyes open and eyes closed data: the lines obtained under eyes open were almost flat whilst those obtained under eyes closed were steep.

Thus there was an apparently different way of scoring the body sway under eyes open and eyes closed, though not under feet apart as opposed to feet together conditions. The same conclusion was reached with a different analysis, when the scores given for areas lying within the same range were compared across visual conditions. Taking into consideration an overlapping range of eyes open and eyes closed areas (range $300-1000 \mathrm{~mm}^{2}$, mean eyes open $=502.8 \mathrm{~mm}^{2}$ and mean eyes closed $=577.4$ $\mathrm{mm}^{2}$, not significantly different) and of the corresponding scores (range 10-5.75, mean eyes open 8.85 and eyes closed 7.95), it was found that a significantly higher score $(\mathrm{p}<0.0001)$ was assigned to similar sways when vision was allowed as opposed to when it was denied. Therefore, it would be prudent to conclude that scoring is influenced, although to a limited extent, by the visual conditions.

This is also depicted in fig 4 , where the mean scores attributed to all trials (both feet together and feet apart) performed by each subject were plotted against the corresponding mean sways. The slope of the lines best fitting the data points was significant for both eyes open $(\mathrm{p}<0.01)$ and eyes closed $(\mathrm{p}<0.0001)$. Further, the eyes open data points (A) lay above the eyes closed points $(B)(p<0.05)$ and showed less scatter than the eyes closed points for similar changes in sway. It can be seen that (1) on average, subjects with large sways scored their performance lower than did subjects with small sways; (2) the error bars of scores and sways did not depend either on the position in the plot of the respective data points (large mean sway and small mean score or vice versa) or on the visual condition.

The output of the platform also included, as said above, the value of the sway path travelled by the centre of foot position during the performance of the stance trial. Therefore, for each trial and subject, the stability score was also referenced to the sway path, on the hypothesis that subjects' evaluation of body sway would be better correlated with sway path than sway area. However, the overall relation described for the sway area was substantially the same for the sway path; both the logarithmic relation, and the $\mathrm{R}^{2}$ coefficient of the lines best fitting the transformed data were similar.

ELDERLY CONTROLS

The elderly normal subjects yielded sway areas not significantly larger than those of the young subjects under eyes open conditions (fig 5); with eyes closed, elderly subjects swayed to a slightly larger extent, both with feet apart and feet together $(\mathrm{p}<0.05)$. The scores were accordingly slightly lower. Also in this group, the relation between average scores and sway areas was logarithmic (fig 6). Again, the slope
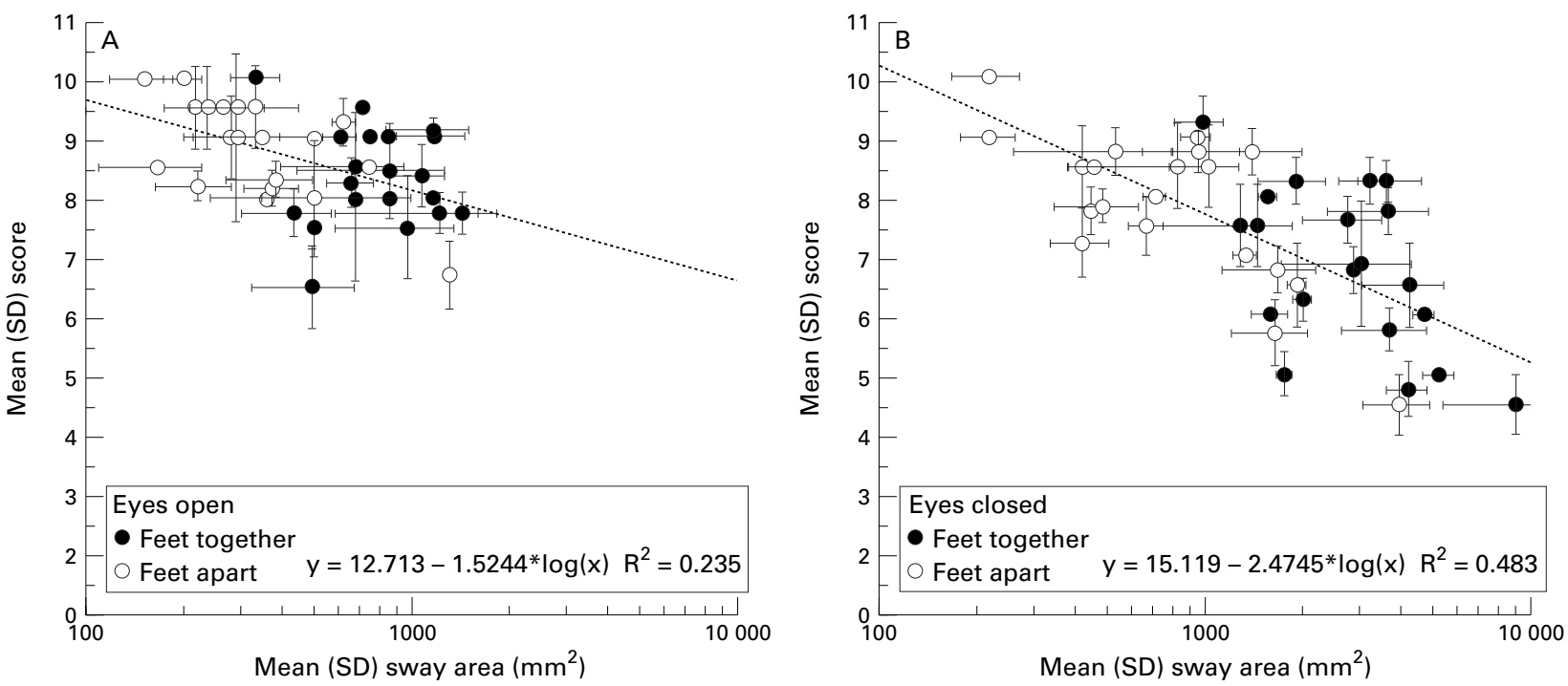

Figure 6 Relation between mean score and mean sway area in the elderly subjects. The lines best fitting the data points of elderly and young subjects (fig 3) obtained in the corresponding visual conditions are not significantly different. 

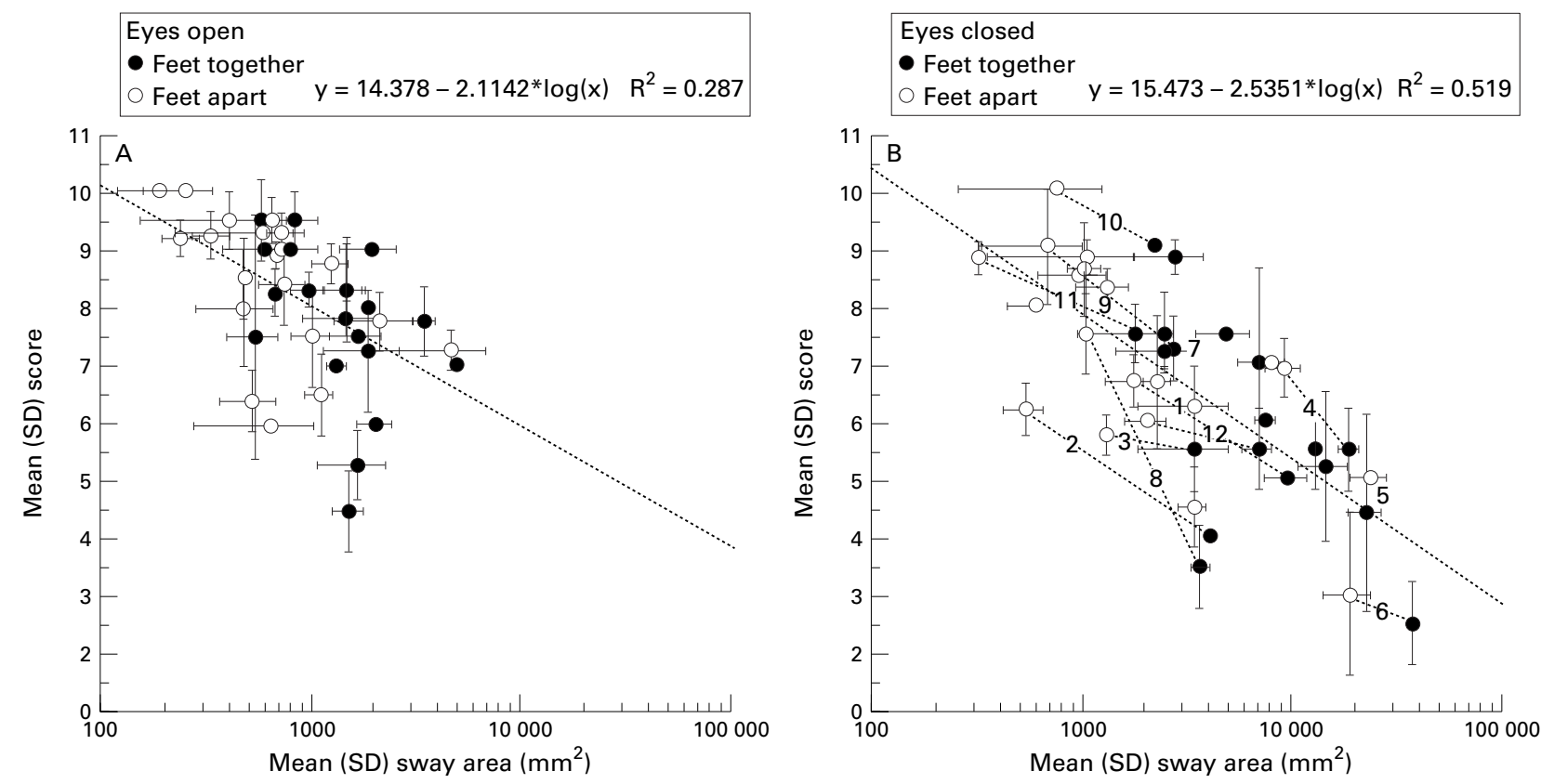

Figure 7 Relation between mean score and mean sway area in the neuropathic patients. In B, dotted segments connect both points pertaining to the same patient; the number on the segment corresponds to the patients' number in table 2. The slopes of both eyes open and eyes closed best fit lines are significantly different from zero, but there is no significant difference in slope between the two lines.

of the lines best fitting the transformed data was significant for both the eyes open $(p<0.01)$ (A) and eyes closed $(\mathrm{p}<0.0001)$ condition (B), and the eyes open data points lay above the eyes closed ones $(0.1>p>0.05)$. The lines best fitting the elderly subjects' eyes open and eyes closed data were not significantly different from the corresponding lines drawn through the young subjects' data, indicating a common mode of subjective sway evaluation.

NEUROPATHIC PATIENTS

The sway areas recorded in these patients were, on average, significantly larger than in both groups of normal subjects, for all stance and visual conditions $(\mathrm{p}<0.0001)$ (fig 5). On average, the scores were much lower than in the normal subjects. There was a progressive decrease in the score with the increase of sway (fig 7), and, again, the relation between average scores and sway areas was logarithmic. In all patients except one, the subjective score worsened with the increase in sway. This is indicated in fig $7 \mathrm{~B}$ (only for 12 patients, for clarity) by a dotted segment connecting the points obtained under both feet apart and feet together postural conditions, where each segment is marked by the number corresponding to the patients' number in table 1 . For one subject, the feet together point was not
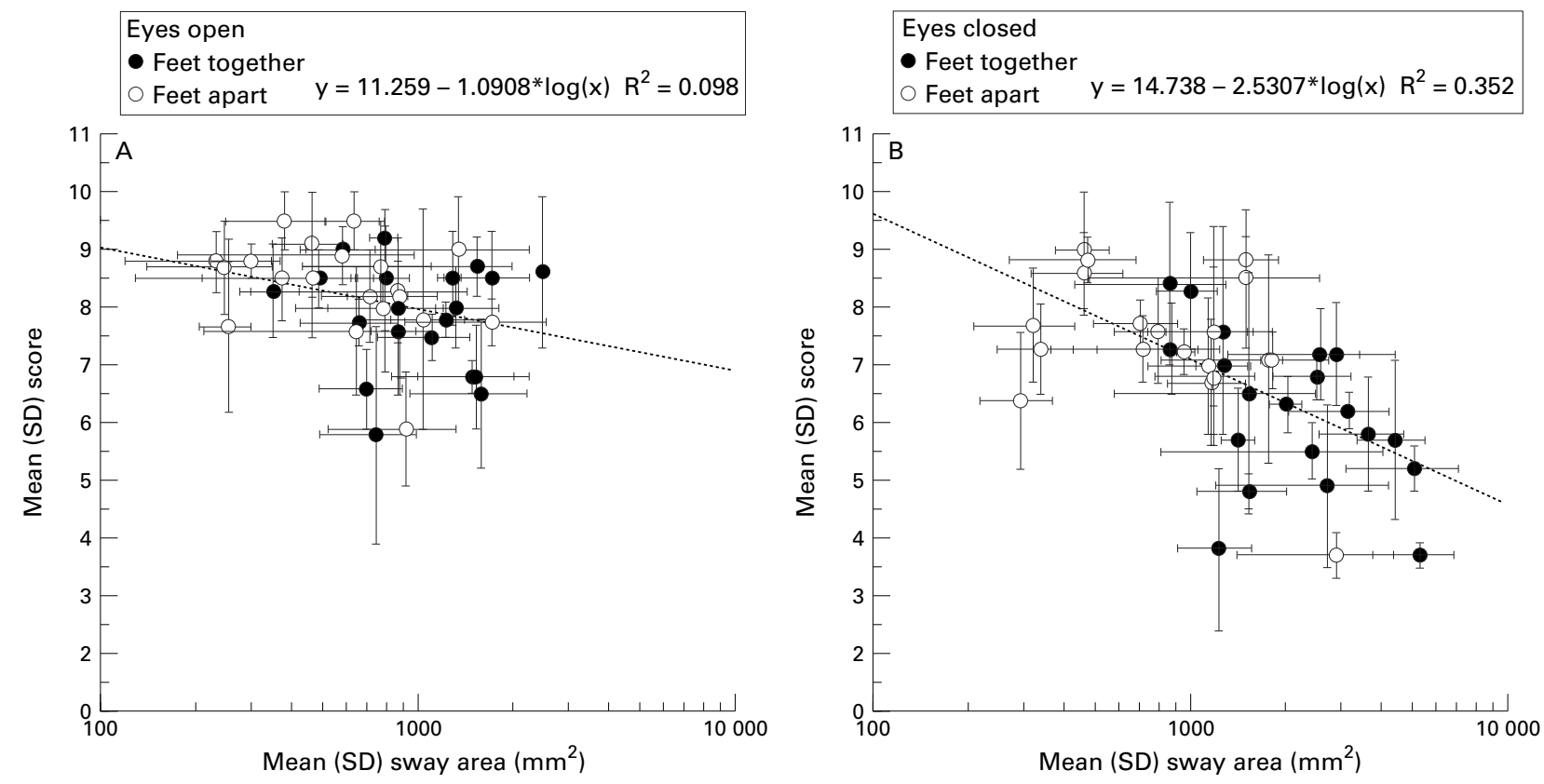

Figure 8 Relation between mean score and mean sway area in the parkinsonian patients. The lines best fitting the data points of parkinsonian patients and elderly subjects (fig 6) obtained in the corresponding visual conditions are not significantly different. 
obtained because the feet together condition could not be maintained for the whole trial. The slope of both best fitting lines was significantly different from zero (eyes open, $\mathrm{p}<0.001$; eyes closed, $p<0.0001$ ), but there was no difference between the slopes of the two lines. The line fitted through the eyes closed data points was almost superimposable with that of the elderly subjects, whereas that fitted through the eyes open data was steeper, though not significantly so, than the corresponding line drawn for the elderly subjects.

\section{PARKINSONIAN PATIENTS}

As shown previously, ${ }^{2}$ the sway areas recorded in the patients with Parkinson's disease were, on average, not larger (or just significantly so under eyes open and feet apart conditions) than those recorded in elderly subjects (fig 5). Furthermore, the scores given by the patients were, on average, similar to those given by the elderly subjects. Within each patient, the subjective score worsened with the increase in sway occurring when the base of support was reduced or when vision was excluded. When the data were represented as in the case of the other three groups, there was an analogous progressive decrease in the score with the increase of sway (fig 8), and the relation between average scores and sway areas was logarithmic. The slopes of the lines best fitting the transformed eyes open and eyes closed data was significantly different from zero (eyes open, $\mathrm{p}<0.05$; eyes closed, $\mathrm{p}<0.0001$ ), and the eyes open data points lay above the eyes closed ones $(p<0.05)$. The best fitting lines were not different from the corresponding lines drawn for the elderly patients. Further, there was no indication of a different behaviour across patients more or less disabled, as the points pertaining to subjects with widely different Webster scores were randomly distributed above or below the best fit lines.

\section{Discussion}

RELATION BETWEEN BODY SWAY AND SUBJECTIVE SCORE

The results show that the scores attributed by each normal subject to the stability of their upright stance reflected the actual sway values recorded by the dynamometric platform. This correspondence was particularly evident under the eyes closed condition, where, regardless of the position of the feet (together or apart) and the consequent large difference in the corresponding sway values, the score closely reflected the magnitude of body sway. Therefore, in their evaluation, subjects were influenced by some net counterpart of the sway itself, regardless of the more or less stable equilibrium conditions connected to the width of the base of support. For the effect of vision, the scores were proportionately less bad during the eyes open than the eyes closed trials for superimposable ranges of body sway. This difference amounted to less than one point on the subjective scale, on average: it seems to be none the less an indication of a more confident state occurring with eyes open. In a sense, therefore, subjects evaluated their posture as more stable when vision was allowed, even with a smaller support base, than with eyes closed. Under both visual conditions, however, the precision of the scoring was similar, as there was no larger scatter of the scores under eyes closed than eyes open, for similar sway area ranges. Conversely, for equal visual conditions, changes in the width of the support base did produce concurrent changes in sway and score. The findings obtained in the elderly subjects basically reproduced those for the young. It would be safe to conclude that age does not seriously impair the procedure for correctly self scoring postural stability, despite the reduced stability limits of the elderly patients with respect to the young subjects ${ }^{32}$ and of the reported difficulties of the elderly subjects in taking advantage of the sensory redundancy in postural control. ${ }^{22}$

Thus normal subjects have the capability of precisely sensing their own sway, independently of sex, age, and constitution. This holds true regardless of the stabilometric variable considered (sway area or sway path); therefore, subjects do not prefer to evaluate the mismatch of the centre of foot pressure from a reference value set at the beginning of the trial (the sway area), with respect to the mean velocity of centre of foot pressure shift (the sway path). This would imply that the subjects' afferent inputs related to both aspects of sway must ultimately be processed in the same way.

Further, different subjects use the same absolute criteria for evaluating body sway, as the mean values of sway and scores of the various subjects lay along the same line despite the considerable scatter of the data. Therefore, subjects do not use as a reference for scoring their "typical" area during a quiet stance, but are able to score their sways according to an absolute criterion. Thus subjects who regularly exhibit large sway areas give low scores, and subjects who regularly exhibit small areas give high scores.

CHARACTERISTICS OF THE SCALE

The use of such a subjective scale, which correlates with numbers entities that are not numbers, ${ }^{23}$ can therefore be tentatively proposed as a criterion for quantifying the self evaluation of body sway. The scale is (1) valid (or unidimensional), as it measures the intended variable; (2) sensitive, as it can evaluate small differences in sway; (3) accurate, as it centres the intended variable within an ample range of it; (4) simple, as its administration to the subjects does not require particular effort in clarifying the question; (5) there would be no harm for the subjects of overemphasising the use of one or the other end of the scale (see below); and (6) it would be easily applicable across laboratories and examiners. ${ }^{24}$ In addition (7) as this scale was actually linear when the sway values were logarithmically transformed, it is tempting to attribute interval properties to it.

The logarithmic relation between the score and the raw area data deserves a comment. This trend might reflect either (1) a reluctance (increasing progressively as sway enlarges) of subjects and patients to give too low a self evaluation, or (2) a real modality of processing the 
afferent information. In the first case, we would assume a scarce compliance with instructions by the subjects, but we have no hints that this was true at the time of the experiment, nor afterwards, when the subjects were interviewed about their attitude. It must also be noted that, when requested to stand under critical conditions for some time (one leg stance), subjects certainly gave very low scores to their performances, without hesitation. We would therefore exclude the idea that subjects' criteria of scoring suffered from any particular tendency such as "end aversion bias" (or "central tendency bias"), whereby extreme scores are less likely to be used than centrally positioned scores, or "positive skew" whereby scores close to maximum are preferred ("ceiling effect") as a self praise..$^{25}$ In the last case (real modality of processing the afferent information), it is remarkable that the relation between the score and the actual body sway most closely resembled the WeberFechner's psychophysical relation $\mathrm{I}=\mathrm{c} \bullet \log$ $\left(\mathrm{S} / \mathrm{S}_{0}\right)$, whereby the capacity for distinguishing between weights depends on the magnitude of the reference weight. In passing, it is interesting to note that, within a certain range, postural sway deviations induced in standing subjects by changes in visual flow have been reported to be proportional to the logarithm of visual motion amplitude. ${ }^{26}$

Subjects were therefore attributing a score to an input related to the mismatch between a reference "optimal" stability and the overall stability of their actual standing within the duration of the trial. In this evaluation, they would have steadily kept in mind the ideal minimum sway for reference, or the "reference frame". ${ }^{27}{ }^{28}$ Of course, the postural performance could be influenced by the cognitive process required of the subjects, ${ }^{29}$ involving at least a judgement about their performance and an operation of their working memory within the period of the trial. However, this does not necessarily imply a competition between the tasks with degradation of either or both. ${ }^{30} \mathrm{It}$ seems reasonable to speculate that the attentional demand required here by the two concurrent tasks did not exceed the subjects' conscious processing capacity. Further, in the subjects who, on the occasion of previous recordings made for other purposes, stood at ease without accompanying cognitive tasks, there was no difference in the average body sway with respect to the present performances. Conversely, the judgement corresponded with the performance, so that the cognitive process itself was unlikely to be impaired.

\section{NEUROPATHIC PATIENTS}

The results obtained in patients with peripheral neuropathies to a large extent confirm the above interpretation, as the logarithmic relation between sway and score was still valid, despite the very large sways and low scores in this group. Therefore, patients were using the same criteria as normal subjects in estimating their sway, as indicated by the slope of the line fitted through the eyes closed data, and were almost as accurate as normal subjects in so doing (compare figs 4 and 7 ). Thus the soma- tosensory input is inadequate to allow a good "reflex" control of posture, as indicated by the large sways of these patients, and seems redundant $^{31}$ as far as sway perception is concerned. Incidentally, we found no correlation between the conduction velocity and sway area in these patients, as already shown by Bergin et $a l .^{32}$ However, in both studies, the only sensory nerve tested was the sural nerve, leaving the possibility that increased sway was related to the degree of impaired proprioception. The fact that reduced sense of vibration was indeed related to the amount of sway, ${ }^{32}$ indicates that afferents such as secondary spindle afferent fibres may be better related to sway control, as suggested elsewhere. ${ }^{33}$

There was an indication of a different behaviour of these patients when tested under eyes open conditions, because the slope of the line fitting the eyes open data was nearly as steep as that fitting the eyes closed data. Apparently, in the neuropathic patients, the fact that the somatosensory input was inadequate resulted in an underscoring of balance, despite allowed visual input. Vision did help to decrease the sway, but was not enough to counterbalance the feeling of unsteadiness. In this connection, the possibility should be entertained that the dissociation between the ability of vision to reduce the absolute amount of body sway and its ability to counterbalance the feeling of unsteadiness may be related to the absolute amount of sway, which was much larger in the neuropathy group than in any other group.

\section{PARKINSON'S DISEASE}

The results obtained in patients with idiopathic Parkinson's disease were closely superimposable with those obtained in normal subjects, indicating that the basal ganglia are not involved in the subjective perception and evaluation of body sway. This finding was rather unexpected if it is considered that basal ganglia receive inputs from multiple cortical regions with different sensory and integrative functions, ${ }^{34}$ and that abnormal basal ganglia activity caused by a nigrostriatal lesion can disrupt aspects of tactile and kinaesthetic perception. ${ }^{35}{ }^{36}$ The present finding suggests, therefore, that the postural insecurity in parkinsonian patients depends exclusively on their impairment in the coordination between posture and movement, ${ }^{37}$ rather than on any mismatch between sway and perception or evaluation of it. In turn, the disturbed coordination between posture and voluntary movement may reflect an inability to appropriately incorporate correctly perceived information in the preparation or execution of movement.

The fact that both normal subjects and parkinsonian patients, and, within limits, sensorially impaired patients, used the same criteria and gave reproducible estimates of sway indicates that such an estimate is a very basic psychophysical process, most likely very important for the everyday fine control of stance, and that the nervous structures involved in the perception and evaluation of body sway are very robust, much more than the structures involved in balance control itself. 
PSYCHOPHYSICAL EVALUATION

The criteria of self scoring postural stability used by the subjects can hardly be reduced to an evaluation of a preferred sensory input, but may depend on the integration of the peripheral information in the subjects' egocentric representation of the space within which their body moves. ${ }^{38}$ The most parsimonious explanation, basically founded on the shape of the relation between score and sway, is that subjects were able to evaluate their sway as a whole, putting into operation the same neural mechanisms that allow us to psychophysically evaluate the intensity of other more "simple" stimuli such as sound or weight. It is not unlikely that, in this process, they would also have evaluated the effort necessary for correcting their posture by moment to moment adjustments of the force level in the antigravity muscles. ${ }^{3}$ It has been hypothesised that, besides operative (reflex) control to compensate deviations from a reference position, the system of postural control includes one additional level, which elaborates this reference using information about mutual positions of body links, muscular torques, and interaction with the support, on the basis of criteria taking into account the energy cost of standing and demands for stability and security. ${ }^{39}$ The reference for the maintenance of orthograde stance is a multimodal construct, which as a rule remains unconscious during quiet stance. It is shown here that subjects can, however, consciously enter this construct when asked to do so, and give a "good" report of what is going on in their brain during the control of their (inherently unstable) orthograde posture.

The afferent and efferent connections of the posterior parietal cortex suggest that this area is ideally placed to perform the complex computations which are necessary to combine the different primary sensory and motor representations to enable us to build up our egocentric spatial frame of reference. ${ }^{39} 40$ Further, not only sensory signals of different modalities but also efference copy signals from motor structures converge in the posterior parietal cortex, ${ }^{41}$ making this area of the brain the most likely candidate for evaluating the oscillations of the body and the efforts produced to minimise it.

Thanks are due to Drs Cinzia Miscio and Fabrizio Pisano for referral of patients. This work was supported in part by a
"Ricerca Corrente" $1997 / 98$ Grant from the Italian Ministry of "Ricerca Corrente" $1997 / 98$ Grant from the Italian Ministry of Health, and by Telethon, Italy, gran
Allpress scrutinised the English.

1 Horak FB, Nutt JG, Nashner LM. Postural instability in

parkinsonian subjects. $\mathcal{F}$ Neurol Sci 1992;111:46-58.
2 Schieppati M, Grasso M, Siliotto R, et al. Effect of age, Schieppati $M$, Grasso $M$, Siliotto $R$, et al. Effect of age,
chronic diseases and parkinsonism in postural control. In: Stelmach GE, Hömberg V, eds. Sensorimotor impairment in the elderly. Dordrecht: Kluwer Academic, 1993:355-73.

3 Schieppati M, Hugon M, Grasso M, et al. The limits of equilibrium in young and elderly normal subjects and in parkinsonians. Electroenceph Clin Neurophysiol 1994;93: 286-98.

4 Schieppati M, Nardone A. Free and supported stance in Parkinson's disease. The effect of posture and postural set on leg muscle responses to perturbations, and its relation to the severity of the disease. Brain 1991;114:1227-44.

5 Richardson JK, Hurwitz EA. Peripheral neuropathy: a true risk factor for falls. $\mathcal{F}$ Gerontol 1995;50:M211-5.

6 Nardone A, Pisano F, Miscio G, et al. Demyelinating neuropathy affects to a different extent the short- and neuropathy affects to a different extent the short- and
medium-latency responses of lower limb muscles to stance medium-latency responses of lower limb muscles to stance
perturbation. fournal of the Peripheral Nervous System 1997; 2:99.

7 Horak F. Clinical assessment of balance disorders. Gait Posture 1997;6:76-84.
8 Wade DT. Measurement in neurologic rehabilitation. Curr Opin Neurol 1993;6:778-84.

9 Tinetti ME, Richman D, Powell L. Falls efficacy as a measure of fear of falling. F Gerontol 1990;45:P239-43.

10 Jacobson GP, Newman CW, Hunter L, et al. Balance function test correlates of the dizziness handicap inventory. 7 Am Acad Audiol 1991;2:253-60.

11 Yardley L, Masson E, Verschuur C, et al. Symptoms, anxiety and handicap in dizzy patients: development of the vertigo symptom scale. F Psychosom Res 1992;36:731-41.

12 Horak FB, Macpherson JM. Postural orientation and equilibrium. In: Rowell LB, Shepherd JT, eds. Exercise: regulation and integration of multiple systems. Handbook of physiology, section 12. New York: Oxford University Press 1997:255-92.

13 Tinetti ME. Performance-oriented assessment of mobility problems in elderly patients. F Am Geriatr Soc 1986;34: $119-26$.

14 Mauritz KH, Dichgans J, Hufschmidt A. Quantitative analysis of stance in late cortical cerebellar atrophy of the anterior lobe and other forms of cerebellar ataxia. Brain 1979;102:461-82.

15 Diener HC, Dichgans J, Bacher M, et al. Quantification of postural sway in normals and patients with cerebellar diseases. Electroenceph Clin Neurophysiol 1984;57:134-42.

16 Tarantola J, Nardone A, Tacchini E, et al. Human stance stability improves with the repetition of the task: effect of feet position and visual condition. Neurosci Lett 1997;228: $75-8$.

17 Portney LG, Watkins MP. Foundations of clinical research. Applications to practice. East Norwalk, Connecticut: Appleton and Lange, 1993:505-28.

18 Walmsley RP, Amell TK. The application and interpretation of intraclass correlations in the assessment of reliability is isokinetic dynamometry. Isokin Exer Sci 1996;6:117-24.

19 Moore PG, Edwards DE. Standard statistical calculations. London: Pitman and Sons, 1965.

20 Armitage P. Statistical methods in medical research. Oxford: Blackwell, 1971:271-84.

21 Nardone A, Siliotto R, Grasso M, et al. Influence of aging on leg muscle reflex responses to stance perturbation. Arch Phys Med Rehabil 1995;76:158-65.

22 Hay L, Bard C, Fleury M, et al. Availability of visual and proprioceptive afferent messages and postural control in elderly adults. Exp Brain Res 1996;108:129-39.

23 Macnaughton RJ. Numbers, scales, and qualitative research. Lancet 1996;347:1099-100

24 Wright BD, Linacre JM. Observations are always ordinal; measurements, however, must be interval. Arch Phys Med Rehabil 1989;70:857-60.

25 Streiner DL, Norman GR. Health measurement scales. A pratical guide to their development and use. New York: Oxford University Press, 1989:54-64.

26 Lestienne F, Soechting J, Berthoz A. Postural readjustments induced by linear motion of visual scenes. Exp Brain Res 1977;28:363-84.

27 Berthoz A. Reference frames for the perception and control of movement. In: Paillard J, ed. Brain and space. New York: Oxford University Press, 1991:81-111.

28 Massion J. Mouvement, posture and equilibrium: interaction and coordination. Prog Neurobiol 1992;38:35-56.

29 Maki BE, McIlroy WE. Influence of arousal and attention on the control of postural sway. $\mathcal{F}$ Vest Res 1996;6:53-9.

30 Kerr B, Condon SM, McDonald SA. Cognitive spatial processing and the regulation of posture. $\mathscr{F}$ Exp Psychol 1985;11:617-22.

31 Peterka RJ, Benolken MS. Role of somatosensory and vestibular cues in attenuating visually induced human postural sway. Exp Brain Res 1995;105:101-10.

32 Bergin PS, Bronstein AM, Murray NMF, et al. Body sway and vibration perception thresholds in normal aging and in patients with polyneuropathy. $\mathcal{F}$ Neurol Neurosurg Psychiatry 1995;58:335-40.

33 Nardone A, Pisano F, Miscio G, et al. Demyelinating neuropathy affects to a different extent the short- and medium-latency responses of lower limb muscles to stance perturbation. Fournal of the Peripheral Nervous System 1997; 2:99.

34 Brown LL, Schneider JS, Lidsky TI. Sensory and cognitive functions of the basal ganglia. Curr Opin Neurobiol 1997;7: 157-63.

35 Demirci M, Grill S, McShane L, et al. A mismatch betwen kinesthetic and visual perception in Parkinson's disease. Ann Neurol 1997;41:781-8.

36 Klockgether T, Borutta M, Rapp H, et al. A defect of kinesthesia in Parkinson's disease. Mov Disord 1995;10: 460-5.

37 Bouisset S. Posturo-kinetic capacity in the disabled. Behav Brain Sci 1996;19:71.

38 Berthoz A, Israel I, Georges-Francois P, et al. Spatial memory of body linear displacement: what is being stored? Science 1995;269:95-8.

39 Gurfinkel VS, Ivanenko YuP, Levik YuS, et al. Kinesthetic reference for human orthograde posture. Neurosci 1995;68: $229-43$

40 Bonda E, Frey S, Petrides M. Evidence for a dorso-medial parietal system involved in mental transformations of the body. $\mathcal{F}$ Neurophysiol 1996;76:2042-8.

41 Andersen RA, Snyder LH, Bradley DC, et al. Multimodal representation of space in the posterior parietal cortex and its use in planning movements. Ann Rev Neurosci 1997;20: 303-30. 